\title{
LA ESTRUCTURA FINA DE LA REVOLUCIÓN QUÍMICA DEL SIGLO XVIII ${ }^{1}$
}

\author{
Anna EsTANY \\ Universitat Autònoma de Barcelona
}

\begin{abstract}
Resumen
La revolución química del siglo XVIII ha sido uno de los casos históricos más estudiados por los filósofos interesados en explicar el progreso de la ciencia. T. Kuhn, I. Lakatos, S. Toulmin y L. Laudan, entre otros, hacen referencia a este cambio en sus modelos de cambio cientifico. La mayorí de los autores hacen análisis globales del paso de la química del flogisto a la química del oxígeno pero descuidan los cambios parciales aunque importantes. El objetioo de este trabajo es el estudio de los cambios ontologicos que se produjeron durante el siglo XVIII, tomando para ello algunas teorias de la referencia a fin de eoaluar la capacidad referencial de la ontologia de la química del flogisto y la de la química del oxigeno. Esto nos permite penetrar en la microestructura de la reoolución química del siglo XVIII y perfilar una parte de su estructura fina.
\end{abstract}

\section{Nueva perspectiva en la explicación de la dinámica científica}

La revolución química del siglo XVIII adquirió especial importancia para los filósofos a partir de la irrupción de la historia en la filosofía de la ciencia en la década de los sesenta, abandonando así

\footnotetext{
1 * Este artículo es una versión revisada de la ponencia presentada en las "Jornades d'estudi de la figura i obra d'A.L. Lavoisier", Barcelona, mayo de 1994.

Este trabajo ha sido realizado en el marco del Proyecto de Investigación "Progreso y modelos de dinámica científica" del que soy investigadora principal (PB92-0846-C06-06).
}

Endoxa: Series Filosoficas, $n^{\circ}$ 7, 1996, UNED, Madrid:

Anna Estany: La estructura fina de la Revolución Quimica del siglo XVIII. pp. 21-42. 
la idea, que había estado presente en todos los autores del Círculo de Viena, de que no es posible una lógica del descubrimiento y que, en consecuencia, la filosofía debía limitarse a la lógica de la justificación. La revolución química constituye un ejemplo paradigmático de cambio científico aducido por todos los autores que han teorizado sobre la dinámica científica. Así, la revolución química es vista por $T$. Kuhn como un cambio de paradigma, por I. Lakatos como un cambio de programa de investigación científica, por S. Toulmin como una revolución conceptual y por L. Laudan como un cambio de tradición de investigación ${ }^{2}$. De estos autores el que de forma específica analiza este caso histórico es Kuhn, aunque los demás también hacen referencia explícita a A.L. Lavoisier como el principal artífice de la creación de la química moderna y el que desbancó la química del flogisto formulada por Stahl.

Desde Kuhn hasta nuestros días se han producido cambios importantes en la forma de acercarse al desarrollo de la ciencia, en el sentido de una preocupación creciente por buscar la estructura fina de las revoluciones científicas, lo cual ha supuesto hacer más complejo el aparato conceptual de los modelos de cambio científico y un análisis más en profundidad de aspectos parciales de dichas revoluciones. Podríamos decir que se han abandonado las aproximaciones holistas en aras de un mayor rigor y detalle en el análisis. Si nos fijamos en las categorías conceptuales del modelo Kuhniano - al menos del primer Kuhn- tenemos sólo el concepto de "paradigma", en cambio Lakatos introduce las categorias de "centro firme", "cinturón protector", "heuristica positiva" "heurística negativa" y "modelos", como entidades que forman parte del "programa de investigación", y Laudan distingue en las "tradicio-

\footnotetext{
${ }^{2}$ Una exposición detallada de la crítica a los modelos de Kuhn, Lakatos y Laudan, y de una propuesta alternativa de explicación de la dinámica científica se encuentra en Estany (1990). Estas críticas y la propuesta de una nueva perspectiva toman como punto de referencia la revolución química del siglo Xvill, y son el resultado de contrastar esta caso histórico con los modelos de Kuhn, Lakatos y Laudan. Aquí me limitaré a exponer los elementos fundamentales de los modelos de dinámica científica según la nueva perspectiva.
} 
nes de investigación" los "problemas", la "ontologia" y la "metodología". Vemos pues que, progresivamente, hay un desarrollo del aparato conceptual con el cual abordar los cambios científicos.

En esta línea de búsqueda de nuevos instrumentos analíticos podemos enumerar la crítica a los modelos de cambio científico propuestos hasta el momento. Por un lado, la maquinaria conceptual que ofrecen dichos modelos es insuficiente para abordar la historia de la ciencia (aunque en este sentido habría que hacer diferencias) y, por otro, no es posible dar cuenta de todos los casos históricos importantes con un sólo modelo, de aquí la propuesta dé tipologías en la explicación de la dinámica científica ${ }^{3}$.

'Por lo que respecta a la maquinaria analítica, las categorías conceptuales que considero ineludibles distinguir en toda secuencia de la historia de una disciplina científica son las siguientes: i) el cuerpo teórico; ii) la ontología del cuerpo teórico; iii) el campo de aplicación; iv) los principios metodológicos; y v) los instrumentos y técnicas. Por lo que respecta a una alternativa al modelo único, la idea es que pueden darse diversos tipos de cambios, por ejemplo, cambios totales en que cambian todos los elementos antes citados y cambios parciales en que cambian algunos de los elementos; también puede haber cambios en que uno de estos elementos sea el motor del cambio; o estructuraciones distintas a partir de los mismos elementos.

Otro aspecto a tener en cuenta en la explicación de la dinámica científica es el que se refiere los criterios para la elección entre teorías ya que, si queremos que los modelos de dinámica científica no sean sólo una reconstrucción de la historia de la ciencia (o lo que es lo mismo, si queremos que la filosofía de la ciencia diacrónica no se limite al aspecto puramente descriptivo de la filosofia de la ciencia) sino también una evaluación epistemológica de los cambios científicos (o lo que es lo mismo, si también queremos poner en juego sus funciones normativas) entonces es imprescindible que en todo análisis filosófico de los casos históri-

\footnotetext{
${ }^{3}$ Para un análisis de las tipologías ver Estany (1994).
} 
cos entre en juego el contexto de la justificación. La idea que está a la base de estas últimas reflexiones es que es imprescincible recurrir al contexto de la justificación si los modelos de cambio científico tienen que dar cuenta del progreso cognitivo de la ciencia, es decir, si hay que evaluar hasta qué punto los científicos se han guiado por fines cognitivos.

A partir de estos presupuestos, la revolución química del siglo XVIII supuso un cambio total, afectando a todos los elementos descritos: cuerpo teórico, metodología, ontologia, instrumentos y campo de aplicación aunque, como dice A. Donovan (Donovan, 1988), hay aspectos como los fundamentos filosófico-metodológicos que han sido desatendidos 0 , al menos, no se han tenido en cuenta cuando, en realidad jugaron un papel muy importante en la crítica a la teoría del flogisto.

La apuesta por abordar todos los aspectos que se ponen en juego en la dinámica científica no implica que nos quedemos sólo en generalidades sino que cualquier modelo debe poder reflejar la complejidad de los procesos científicos. Esto significa que los análisis globales de un cambio científico tienen que complementarse con análisis parciales que nos permitan llegar a las estructura fina de la dinámica científica. Es en la tarea de los análisis parciales donde se inserta el presente trabajo. El objetivo es, pues, la evaluación de los cambios ontológicos que se produjeron en la revolución química del siglo XVIII y para ello voy a tomar algunas teorías sobre la referencia desarrolladas en filosofía de la ciencia, ya que la valoración de la ontología está relacionada con su capacidad referencial.

En primer lugar vamos a explicitar las cuestiones que analizaremos de este caso histórico, en segundo lugar, expondremos algunas teorías de la referencia y las consecuencias que tienen para las cuestiones que queremos analizar, y finalmente, en vista a los resultados anteriores propondremos algunas alternativas a las teorías de la referencia antes citadas que, en opinión de la autora, captan mejor el espíritu y la letra de la revolución química del siglo XVIII. 


\section{La ontología básica y compuesta de la química del flogisto y de la química del oxígeno}

Veamos lo que puede ser considerada la ontología de Stahl, es decir, de qué entidades está compuesta la teoría del flogisto ${ }^{4}$. Stahl habla de cuerpos y materiales de la naturaleza. La terminología está poco determinada y no se caraterizan con precisión los conceptos de "cuerpo", "material" y "substancia", ni la diferencia que hay entre ellos. Las substancias más simples que encontramos en el sistema del flogisto son los principios que están asociados a la primera, segunda y tercera tierra (vitrificable, combustible y mercurial, respectivamente) y es sobre la segunda tierra, correspondiente al principio de inflamabilidad, sobre la que Stahl construye la teoría del flogisto. No es que los cuerpos estén compuestos de estos principios elementales, a modo de elementos primitivos que configuran la composición de los cuerpos, sino que estos principios entran en la composición, pero el cuerpo está compuesto de otros materiales. En el sistema de Stahl no tenemos una ontología delimitada ni reducible a un número de elementos, por tanto, no es posible tener una terminología precisa y lógicamente deducible de un lenguaje primitivo. Cada cuerpo tiene un nombre sin que tenga ninguna relación con los nombres de los demás cuerpos, ni con los nombres de las substancias que los componen.

Veamos lo que dice el propio Stahl sobre este principio-fuego o flogisto en el Tratado sobre el azufre:

La primera cosa a examinar en relación al principio azufroso es: 1. su propiedad relativa al fuego; 2 . la propiedad que tiene de colorear; 3 . su combinación íntima con otras substancias sutiles; 4 . la manera de comportarse en relación al agua y a la humedad; 5 . su sorprendente división y atenuación; 6 . su naturaleza, sea en estado sólido, sea en estado fluido; 7 . dónde se encuentra. (Stahl, Azufre, pp:55-56).

${ }^{4}$ Una exposición más detallada se encuentra en Estany, 1990. 
En realidad, al flogisto se le asignaron propiedades muy diversas: se le consideró terroso, acuoso, elástico por naturaleza, y a veces también como una substancia grasosa, azufrosa, inflamable, oleosa, viscosa etc., sin embargo, como era inapreciable a los sentidos, era difícil de contradecir las diferentes propiedades que se le asignaban.

En cuanto a la forma de llegar a estas afirmaciones podemos decir que la metodología de Stahl era cualitativa. La definición de las substancias se realizaba a través de la descripción de las propiedades y las reacciones se definían describiendo las propiedades antes y después de la reacción. En cuanto a los instrumentos que utilizaba, podemos decir que Stahl no disponía de aparatos de medir y de pesar con precisión.

La ontología en el sistema químico de Lavoisier estaba formada por los elementos de la Tabla Elemental de química, por tanto, la ontología de la química del oxígeno era limitada. La caracterización de las entidades de la ontología estaban fundamentadas en dos aportaciones teóricas importantes: la definición del concepto de elemento y la formulación de una terminología apropiada a partir de los elementos primitivos, uno de los cuales era el oxígeno que lo caracterizaba en los términos siguientes:

El oxigeno es una de las substancias que con más abundancia se encuentra, ya que constituye casi un tercio del peso de nuestra atmósfera y, por tanto, del fluido elástico que respiramos. Es en este inmenso recipiente en el que viven y crecen los animales y los vegetales y es igualmente de donde extraemos principalmente todo el oxígeno que empleamos en nuestros experimentos. (Lavoisier, Oeuvres, t.I, p.143)

Respecto a los principios metodológicos, Lavoisier adoptó una metodología cuantitativa y, al contrario de Stahl, ideó muchos instrumentos como la balanza y el gasómetro para hacer mediciones precisas. El disponer de estos instrumentos le permitió poner en práctica los principios metodológicos cuantitativistas.

Todo este cambio en la ontología venía respaldado por una serie de principios metodológico-filosóficos sobre la importancia 
del lenguaje que Lavoisier había tomado de Condillac, para quien la causa de nuestros errores está en el hábito de juzgar con palabras, cuyo sentido no hemos determinado y el progreso de las ciencias depende enteramente del progreso de sus lenguajes ${ }^{5}$. La influencia de Condillac llevó a Lavoisier a decir que un lenguaje bien hecho no permitiría a aquellos que ejercen de químicos desviarse de la marcha de la naturaleza. Así pues, uno de los métodos de la nueva nomenclatura era que los nombres de los compuestos se basaran en los nombres de los elementos que las constituían.

\section{El problema del significado de los términos científicos}

Continuamente, tanto en la vida cotidiana como en la actividad investigadora, tomamos compromisos ontológicos y para que estos compromisos sean auténticos y firmes es necesario que las categorías ontológicas posean un grado razonable de claridad, precisión y aplicabilidad (Moulines, 1982: 327). A la hora de calibrar el progreso cognitivo comparando las ontologías de dos secuencias de un período histórico, lo que nos interesa es definir los indicadores epistemológicos de claridad (bien definidos), precisión (instrumentos disponibles) y aplicabilidad (relación con el mundo). Es decir, tenemos que especificar cuáles son los criterios para afirmar que unos términos están mejor definidos, son más rigurosos y reflejan mejor el mundo que otros. Esta idea que puede parecer trivial plantea muchos problemas filosóficos algunos de los cuales vamos a analizar a continuación.

Uno de los problemas filosóficos relacionados con la ontología procede de la relación entre lenguaje y mundo. Si bien, originariamente, era un problema de la filosofía del lenguaje se ha visto que también afecta a la filosofía de la ciencia, ya que los científicos expresan sus teorias en el lenguaje natural además de las formula-

\footnotetext{
${ }^{5}$ Afirmaciones de Condillac citadas por Crosland, 1978, página 171.
} 
ciones matemáticas. Es fundamental para el progreso cognitivo de la ciencia determinar cómo los términos utilizados ("planeta", "gen", "elemento químico" o "clase social") adquieren significado, y cómo fijan su referencia. Si pensamos que la ontología de una teoría está formada por las entidades sobre las cuales se construyen todas las teorizaciones, puede comprendense la importancia de la especificación de la referencia de estos términos para valorar la teoría en su totalidad.

El plantear el problema filosófico de la relación entre lenguaje natural y mundo, es decir, del fundamento para afirmar que ciertos elementos lingüísticos se hallan en relación constitutiva con ciertos elementos extralingüísticos, nos lleva al problema del significado de los términos científicos y, por tanto, a analizar las teorías de la referencia.

\subsection{G. Frege y las categorias semánticas}

Desde Frege sabemos que el lenguaje tiene dos funciones semánticas: referirse a las entidades y señalar los sentidos. Frege pensó que la referencia de una expresión lingüística no agota su significado, es decir, varias expresiones pueden tener la misma referencia pero distinto sentido. El objeto al que una expresión se refiere (o designa, o denota) es su referencia y la peculiar manera de referirse a él es su sentido. El ejemplo típico de Frege es que las expresiones "el lucero de la mañana" y uel lucero de la tarde" tienen la misma referencia: "el planeta Venus», pero distinto sentido.

Los objetos son independientes de su "representación", que es la imagen que la mente se construye de este objeto. A diferencia de la representación, el sentido, es decir, la forma de referirse al objeto, es objetivo, porque puede ser propiedad común de muchos $y$, por tanto, no es parte o modo de la mente individual (Frege, 1971:54). El sentido es el medio intersubjetivo de acercarse a la realidad, una realidad objetiva, pero que no hay que confundirla con la realidad misma. 
Frege, al igual que R. Carnap y A. Tarski, mantiene el punto de vista llamado "semántica referencial», según el cual el valor de verdad de un enunciado se determina a partir de las denotaciones de los nombres que lo componen y de las extensiones de los predicados que lo componen, y ésta es la única noción de verdad y falsedad que se acepta. Los valores de verdad son por lo tanto los objetos a los que refieren las oraciones enunciativas. Se da la circunstancia de que todas las oraciones verdaderas designan lo mismo, lo verdadero, y que igualmente sucede con las falsas que refieren a lo falso. En el enunciado, la predicación se efectúa de un objeto, no de un nombre, y si el objeto referido no existe, entonces la predicación no se produce y no es posible adscribir al enunciado un valor de verdad. Frege afirma que la creencia de que un enunciado es verdadero o falso supone la creencia de que las expresiones nominales incluidas en él designan objetos 0 , dicho de otro modo, que del enunciado, verdadero o falso, se sigue la afirmación de que existe lo referido por sus expresiones componentes.

3.2. La relatividad ontológica y la inconmensurabilidad de las teorías científicas

El sistema fregeano ha recibido críticas por parte de filósofos del lenguaje y filósofos de la ciencia. Por lo que respecta a la dinámica científica es especialmente pertinente la de Quine con sus tesis de la «inescrutabilidad de la referencia" y de la "indeterminación de la traducción». La tesis de la inescrutabilidad de la referencia dice que no hay forma de saber a lo que se refiere un término singular de un lenguaje ni de determinar la verdad de los predicados, al menos de determinarlas (la referencia de un término y la verdad de los predicados) a partir de la conducta tanto real como potencial, y Quine piensa que la conducta es todo lo que importa para cuestiones como el significado y la comunicación. Por 
ejemplo, cuando me comunico con otro individuo que habla mi mismo lenguaje, doy por supuesto que con las mismas palabras nos referimos a lo mismo, pero nunca puedo llegar a confirmar esta hipótesis, ya que la mera aquiescencia u otros síntomas conductuales son insuficientes para determinar la referencia de una expresión. Esto significa que para fijar la referencia de un término hay que hacerlo en función de la referencia de otros términos, es decir, los términos fijan su referencia dentro de un marco lingüístico, pero no existe un punto de vista supralingüístico desde el que poder fijar la referencia de cada término con independencia de sus relaciones con otros términos. La tesis asociada a la inescrutabilidad de la referencia es la de la indeterminación de la traducción, que dice que la traducción de un lenguaje a otro puede hacerse de formas divergentes, todas compatibles con la totalidad de las disposiciones del habla, aunque incompatibles unas con otras (Quine, 1960:27). Por tanto, cualquier traducción siempre es indeterminada.

Ahora bien, si esto es así, si Quine tiene razón entonces la tesis de la inconmensurabilidad de Kuhn tiene un buen argumento a su favor, pero con la tesis de la inconmensurabilidad tenemos pocas y pobres razones para predicar cualquier tipo de progreso científico y nos situamos en el filo de la navaja, al borde de caer en el más puro relativismo. ¿Son inevitables estos pasos, desde la inescrutabilidad de la referencia al relativismo? Yo diria que es dificil pararse en uno de los eslabones, sin embargo, hay que señalar que hay autores como U. Moulines (Moulines, 1991: 137-152) que se paran en el último, es decir, no aceptan el relativismo aún aceptando la tesis de Quine y la de Kuhn. La forma de mantenerse al borde del precipicio relativista pero asegurándose, al mismo tiempo, de no caer nunca por él es -al menos en el caso de Moulines - tomar la metodología como el elemento que trasciende el propio paradigma 0 , lo que es lo mismo, el propio lenguaje, pero entonces la tesis de la inconmensurabilidad queda tan sumamente debilitada que bien poco se 
parece a la formulación original de Kuhn y, sobre todo, a las consecuencias que el propio Kuhn extrajo.

4. Consecuencias de las diferentes teorías de la referencia sobre la revolución química

La óptica desde la que nos situemos no es baladí a la hora de enjuiciar la química de Stahl y la de Lavoisier. Respecto a la ontología de Stahl podemos plantearnos las preguntas siguientes: ¿cuáles son las propiedades de este principio (el flogisto) que forma parte de todos los cuerpos?, ¿cuáles son los modos de referencia del flogisto, es decir, cuál es su potencial referencial?, ¿a través de qué métodos (tanto técnicas como principios metodológicos) se fija la referencia?, ¿hasta qué punto se ponen en juego los criterios de precisión, rigor y aplicabilidad?, ¿qué valoración epistémica podemos hacer de la ontología de Stahl y qué repercusiones puede tener para la valoración de su obra en general?

Según la concepción denominada "semántica referencial" en la cual se incluye Frege y según la cual todas las expresiones que tienen términos no referenciales son falsas o carecen de valor de verdad, todos los enunciados de la teoría del flogisto carecerían de referencia ya que no hay forma de saber a qué se refiere Stahl con el flogisto. Respecto al sistema de Lavoisier no habría problema excepto para los enunciados que incluyeran el término "calórico", que tampoco tiene referencia.

¿Cuál serían las consecuencias a la hora de valorar la obra de J. Priestley y J. Cavendish? Pues difícilmente podría asignarse referencia a los enunciados en que formularon sus investigaciones ya que también el término "flogisto" entra en ellos. Pensemos que desde el enfoque de la semántica referencial, si el "flogisto" no tiene referencia todos los enunciados en los que se encuentre el

\footnotetext{
${ }^{6}$ Priesley y Cavendish fueron dos químicos contemporáneos de Lavoisier que hicieron importantes aportanciones al desarrollo de la química pero interpretaron sus descubrimientos desde la teoría del flogisto.
} 
término "flogisto" son falsos. El argumento discurriría en los términos siguientes: Priestley se sitúa en la comunidad científica flogistonista que fija su referencia como "la substancia emitida en la combustión". Como sabemos que en la combustión ninguna substancia es emitida, todos los enunciados construidos a partir del flogisto tienen que ser falsos o, simplemente, carecen de valor de verdad. Lo mismo ocurriria con términos técnicos como "aire flogisticado" y "aire desflogisticado" que fijan su referencia utilizando el término flogisto.

Otro de los problemas procede de las tesis de Quine y Kuhn, por un lado, y Quine, Kuhn y Moulines, por otro. Con el primer bloque no hay forma de fundamentar el progreso llevado a cabo desde Stahl hasta Lavoisier, no hay forma de dar sentido, o al menos de encontrar algun fundamento a la intuición de que Lavoisier hizo avanzar la química. Con el segundo bloque, es decir, con el salvavidas metodológico de Moulines, no podemos fundamentar la aplicabilidad, entendida ésta como su relación con el mundo, pero sí puede dar cuenta de la precisión y rigor de los términos utilizados.

5. La alternativa de $\mathbf{H}$. Field, su utilización por $\mathbf{N}$. Jardine y $\mathbf{P}$. Smith y las implicaciones de estos trabajos para los enunciados de la teoría flogistonista

H. Field (Field, 1973) presenta una alternativa a la conçepción de la semántica referencial que socava algunas de las críticas de Quine a dicha concepción. El concepto clave de Field es el de "denotación parcial" o "referencia parcial"7. Field critica la concepción de la semántica tradicional porque cree que hay enunciados con valores de verdad que, en cambio, contienen nombres y predicados indeterminados referencialmente $y$, por tanto, tiene sentido preguntar si el enunciado es verdadero o falso

7 Field utiliza indistintamente "denotación" y "referencia". 
aunque no lo tenga el preguntar qué denota el nombre o cuál es la extensión del predicado. Pero también critica a Quine porque, aunque admite que hay términos que están referencialmente indeterminados no cree que pueda sacarse la conclusión de que no hay una relación objetiva entre los términos, por un lado, y las entidades extralingüisticas, por otro.

La reformulación que hace Field de la teoria de la referencia de Frege y la recuperación de una parte de la tesis de Quine, introduciendo al mismo tiempo el concepto de referencia parcial, es tomada por algunos teóricos del cambio científico como punto de partida para el análisis filosófico del progreso cognitivo de la ciencia. Tal es el caso de N. Jardine (Jardine, 1978) y P. Smith (Smith, 1981) cuyas tesis vamos a presentar a continuación de forma sucinta. Jardine y Smith plantean el problema de cómo dar sentido a las teorías científicas que nos preceden y que, a primera vista, no tienen ningun valor de verdad porque sus términos no tienen referencia y sus predicados no tienen extensión. Su postura estaría fuera de la semántica referencial y también de la inescrutabilidad de la referencia. Smith (1981:449) no pretende mostrar que todos los términos, incluso dentro del propio lenguaje, poseen una referencia escrutable sino que no hay razones para mantener, tal como hace Quine, que se dé la inescrutabilidad a gran escala, y concluye que el argumento de Quine fracasa en mostrar que no hay una relación de referencia determinada entre expresiones de un lenguaje y partes del mundo.

Para salvar el escollo de la semántica referencial Smith (Smith, 1981) distingue dos formas de abordar las aportaciones de nuestros predecesores: i) una es pensar que los predicados utilizados fallan porque no tienen extensión de acuerdo a nuestro esquema conceptual; ii) otro que dichos predicados tienen extensión pero expresan falsas creencias sobre lo que se aplican. El caso del flogisto estaría en el caso i). Sin embargo, Smith no saca las consecuencias de que todos los enunciados son falsos. El siguiente paso en su argumentación es distinguir dos cuestiones: a) ¿cuál es 
la extensión del predicado "alfa"?; b) ¿tuvieron éxito los miembros de la comunidad $C$ en describir algo cuando utilizaron "alfa"? Sustituyendo alfa por flogisto y $\mathrm{C}$ por la comunidad científica en torno a Stahl, Smith (siguiendo a Jardine) analiza el enunciado siguiente, plausible en el marco de la comunidad flogistonista: "añadiendo ácido de vitriolo al granulado de zinc se observó un desprendimiento de flogisto".

Para nosotros - sigue Smith- es obvio que lo que el químico observó es el desprendimiento de hidrógeno al añadir ácido sulfúrico al granulado de zinc. No distinguir entre las dos cuestiones nos lleva a que no podamos asignar ningún valor de verdad a los enunciados de la teoría del flogisto y además parece un milagro que la comunidad de químicos lograra un acuerdo sobre un predicado que no tenía ninguna aplicación. Podemos decir que habia algunas instancias del flogisto tal como era utilizado por los quimicos del siglo XVIII que pueden ser atribuidas a lo que nosotros llamamos hidrógeno. Desde el punto de vista de Field, el flogisto parcialmente se refería al hidrógeno $y$, parcialmente también, a muchas otras substancias. Lo que tenemos es una disyunción de todas aquellas entidades a las que parcialmente refería.

A medida que nos vamos acercando a los flogistonistas más próximos a Lavoisier se van reduciendo los términos de esta disyunción y la capacidad referencial aumenta.

\section{P. Kitcher : valoración cognitiva de las aportacionẹs de J. Priestley y J. Cavendish}

Así como Smith y Jardine se centran en el problema de cómo dar sentido a los enunciados con términos que no tienen referencia, Kitcher analiza de forma más pormenorizada casos fronterizos como los trabajos de Priestley y Cavendish. Su idea es que el progreso científico supone un cambio conceptual, que éste es gradual y que se mide en función de su capacidad referencial. Esto le lleva a la teoría de la referencia de Frege, a las críticas de $\mathrm{H}$. 
Putnam (Putnam, 1975) y S. Kripke (Kripke, 1980) y a la concepción de Field.

Kitcher mantiene que las críticas de Kripke y Putnam a Frege están basadas en un supuesto sobre los modos de referencia que podría ser abandonado. Segun Kitcher, podemos partir de dos suposiciones distintas respecto a los modos de referencia: (1) que haya un modo de referencia uniforme para todas las ocurrencias de un término-tipo; (2) que un término científico (término-tipo) tenga un referencial potencial heterogéneo. $Y$ sostiene que los problemas que Putnam y Kripke ven en el sistema fregeano proceden de suponer (1), pero si suponemos (2) y reformulamos la noción de "sentido" con la noción de "modos de referencia", tal como lo entiende Field, estos problemas desaparecen.

Otro de los puntos problemáticos del sistema fregeano es que los sentidos son entidades intencionales, es decir están en función de lo que el hablante quiere decir con la expresión determinada. Pero Kitcher le da un enfoque naturalista a los sentidos de Frege. Para ello recurre a la ciencia cognitiva para explicar cómo adquirimos el potencial referencial de un término, que consiste en incorporarar un conjunto de propensiones a nuestra memoria procesal. Esto supone que los sentidos ya no son entidades abstractas sino procesos psicológicos situados en nuestro aparato cognitivo, con lo cual tenemos un modo natural de acercarnos al sistema fregeano ( Kitcher, 1993;79)

Una vez superados los problemas del sistema fregeano, Kitcher define los criterios que determinan la capacidad referencial de los términos científicos. El concepto clave en el proyecto de Kitcher es el de "potencial referencial" de un término que consiste en el compendio de los "modos de referencia" de dicho término. $Y$ los modos de referencia son las formas de cómo fijamos la referencia, es decir, aquello que hace que una ocurrencia determinada se refiera a un objeto. En este sentido podríamos decir que los modos de referencia equivalen en Frege a la cuestión de cómo fijamos los sentidos de una expresión lingüística.

Con esta concepción sobre la forma y capacidad de fijar la 
referencia, Kitcher mantiene la tesis de que los cambios conceptuales que han causado mayor impacto $y$ han significado mayor progreso cognitivo son aquellos en los que ha habido una reorganización de los conceptos que ha implicado un perfeccionamiento en los potenciales referenciales de los términos clave (Kitcher, 1993:96). Con estos elementos define el progreso científico en los términos siguientes:

(PC) Una práctica P2 es conceptualmente progresiva respecto a una práctica $\mathrm{P} 1$ en caso de que haya un conjunto $\mathrm{C} 2$ de expresiones en el lenguaje de P2 y un conjunto $\mathrm{C} 1$ de expresiones en el lenguaje de P1 tales que:

(a) excepto para las expresiones en estos conjuntos, todas las expresiones que se encuentran en un lenguaje se encuentran en ambos con un referencial potencial común

(b) para cada expresión e en $\mathrm{C} 1$, si hay un género al que algunas ocurrencias de e se refieren, entonces hay una expresión $e^{*}$ en $C 2$ que tiene ocurrencias que se refieren a este género

(c) para cada $e, e^{*}\left\{\right.$ como en (b) \}, el potencial referencial de $\mathrm{e}^{*}$ refina el potencial referencial de e, bien añadiendo una descripción que selecciona el género pertinente, bien abandonando una forma de determinar la referencia que pertenece al potencial referencial de e que ha fracasado en seleccionar el género pertinente. (Kitcher, 1993:104-105)

Esta maquinaria conceptual le sirve a Kitcher para dar razón de la importancia y del papel que jugaron Priestley y Cavendish en la consolidación de la revolución química. Los escritos de los últimos flogistonistas abonan la idea de que encontramos doctrinas verdaderas intentando escapar de un lenguaje que habia fracasado por no tener la capacidad de referir.

Lo interesante de la idea de Kitcher para penetrar en la microestructura de la ontología teórica es la posibilidad de tener un abanico de modos de referencia y la posibilidad de que un término científico tenga un potencial referencial heterogéneo, lo cual supone la posibilidad de que al comparar varias secuencias de la historia de una disciplina algunos modos de referencia se 
solapen, lo cual explicaría el cambio gradual que es, precisamente, lo que ocurrió en la revolución química si nos atenemos a la evolución que sufrió desde Stahl a Lavoisier.

Si nos centramos en el caso de Priestley, vemos que éste fijó la referencia de "aire desflogisticado" ${ }^{8}$ de modos diversos, es decir, de forma heterogénea: por un lado, describiendo este gas como "la sustancia obtenida cuando la sustancia emitida en la combustión es separada del aire", por otro como "la sustancia que Priestley y el ratón respiran". En este caso se ve claro que Priestley proporcionó información que podemos considerar como verdadera aunque él utilizara un lenguaje equivocado. ¿Qué podemos decir de la posibilidad de traducción de los términos de Priestley a los de Lavoisier? Kitcher argumenta en los términos siguientes: si la traducción tiene que preservar los potenciales referenciales, tienen razón Quine y Kuhn de que no es posible la traducción, ya que no hay ningun término del lenguaje de la química posterior a Lavoisier que tenga los mismos potenciales referenciales del término "aire desflogisticado". La falta de ajuste entre los potenciales referenciales de ambas químicas encaja con la idea de Kuhn (Kuhn, 1983) de que los flogistonistas y los químicos modernos clasifican y "ven" el mundo de forma distinta, sin embargo esto no significa que tanto Lavoisier como los químicos actuales no puedan entender las afirmaciones de Priestley y Cavendish, ya que pueden reconocer los potenciales referenciales de los términos que utilizaron Priestley y Cavendish. No sólo podemos comprender lo que Priestley dijo, sino que podemos comprobar que algunos de los enunciados que propuso son verdaderos y podemos explicar cómo mejorar su lenguaje. Lavoisier realizó un avance conceptual revisando los potenciales referenciales e introduciendo el término "oxígeno". En general el refinamiento puede ser, bien por añadir una descripción que identifica el tipo pertinente, bien por abando-

\footnotetext{
"Es importante señalar que aqui "aire desflogisticado" se refiere a la sustancia que Priesley y el ratón respiran, es decir, oxigeno. Las mismas reflexiones podríamos hacer de Cavendish cuando dice que la combinación de "aire inflamable" (hidrógeno) y "aire desflogisticado" (oxígeno) da lugar a agua.
} 
nar una determinación de modo de referencia que pertenece al potencial referencial que ha fallado en identificar el tipo pertinente. En el caso de Lavoisier su innovación consistió en la segunda posibilidad, abandonando así la forma de referir "con desprendimiento de flogisto" porque pertenecía al potencial referencial del "flogisto", pero guardando la expresión "aquello que el ratón y los humanos repiran" y este modo de referencia era conjunto a "aire desflogisticado" y a "oxígeno".

El análisis de Kitcher contribuye a resolver el rompezabezas sobre los lenguajes de los protagonistas de la revolución química, a partir de la tesis de que el cambio conceptual consiste en un cambio en el potencial referencial. Hay que señalar, sin embargo, que el cambio conceptual es sólo un aspecto del cambio científico global, por tanto, deberíamos añadir que el progreso conceptual no agota el progreso científico.

\section{Conclusiones}

El análisis de la estructura fina de las diferencias entre la química de Stahl y la de Lavoisier podría parecer irrelevante en el sentido de que con análisis más a grosso modo, como los realizados con modelos como el de Kuhn o el de Lakatos también quedaría patente la importancia del paso de la química del flogisto a la química del oxígeno. ¿Qué nos aporta el introducirnos en la microestructura de los cambios científicos? En primer lugar, que incluso los cambios más paradigmáticos de los que nadie duda su trascendencia histórica, el análisis de su estructura fina nos proporciona un conocimiento más profundo de este período histórico, del mismo modo que en el campo científico la posibilidad de adentrarnos en el nivel "micro" siempre supone ganar en información $y$ en potencial explicativo de los fenómenos que queremos explicar.

Pero hay otras cuestiones que se clarifican al tener en cuenta diversas concepciones sobre la referencia. Todos tenemos la intuición de que Lavoisier hizo progresar la química pero hay 
concepciones sobre la referencia que no recogen, o recogen mal, esta intuición. He dicho que incluso el modelo de Kuhn, a pesar de su imprecisión, recogía este cambio, sin embargo, si nos introducimos en el micronivel se nos plantean algunos problemas. Por ejemplo, es difícil argumentar a favor del progreso de la química si tomamos en serio la tesis de la inconmensurabilidad, que no es más que la consecuencia inmediata de las tesis de Quine (de la inescrutabilidad de la referencia y de la indeterminación de la traducción). Si no hay criterios (y tal como Kuhn entiende la inconmensurabilidad no los hay) que trasciendan al propio paradigma es imposible decir qué teoría es la mejor.

Por otra parte, si no hay ningun tipo de relaciones objetivas entre términos, por un lado, y objetos extralingüisticos, por otro, entonces ¿cuál es la diferencia entre el flogisto y el oxígeno respecto a su capacidad referencial?. Según Quine, las relaciones semánticas de referencia y significación son "relativas al esquema conceptual", es decir, relativas a la teoria del flogisto y a la teoría del oxígeno, y esto nos lleva a concluir que los términos de dos teorías no pueden ser comparados objetivamente respecto a sus propiedades referenciales. En este punto es pertinente la alternativa de Field en el sentido de que, al mismo tiempo que reconoce que muchos términos científicos están indeterminados referencialmente (de forma absoluta) pueden, sin embargo, referir parcialmente. Por esto, rechaza totalmente las conclusiones a las que llega Quine sobre la falta de relaciones objetivas entre términos y entidades extralingüísticas, lo que permite comparar la capacidad referencial de las teorías.

También estos nuevos enfoques dan cuenta de la comunicación que a finales del siglo XVIII existía entre flogistonistas y antiflogistonistas. Un hecho que prueba esta comunicación es toda la cuestión sobre la nomenclatura química. Crossland (Crossland, 1978 :204) señala que aunque Priestley no habia aceptado la nueva nomenclatura porque era antiflogistonista, se lamentaba porque se había visto forzado a aprenderla para seguir el desarrollo de la 
química y para leer los trabajos de los químicos que sí habían adoptado la nueva nomenclatura. Era habitual a finales del siglo XVIII la publicación de listas de sinónimos, y en el caso de la farmacología era prácticamente obligado ya que dependía la vida de mucha gente. Una muestra de esta comunicación la tenemos en el escritor inglés T. Cavallo que usaba expresiones como "the dephlogisticated or oxygen air" (Crossland, 1978:205).

Hay otras cuestiones en que el llegar o no a la estructura fina de la dinámica científica supone el detectar o no el cambio. Tal es el caso de lo que supusieron para el desarrollo de la química las aportaciones de Priestley y Cavendish. Ambos fueron flogistonistas hasta el final, por lo tanto, desde el modelo de Kuhn deberiamos concluir que pertenecen al paradigmo flogistonista y que viven en un mundo distinto del de Lavoisier, que sus teorías son inconmensurables con las de Lavoisier. Estas afirmaciones creo que serían difíciles de aceptar por cualquier historiador de la química ya que restan todo mérito a estos dos químicos contemporáneos de Lavoisier que, aunque flogistonistas, hicieron importantes aportaciones, y que, sin embargo, desde un análisis a grosso modo quedan bajo el rótulo "teoría flogistonista" un paradigma en franca decadencia. Vemos, pues, la necesidad de tener unos modelos de dinámica científica que tengan el aparato conceptual suficiente para captar la complejidad de la dinámica científica y detectar todos los cambios pertinentes para el desarrollo y progreso de una disciplina científica. En nuestro caso concreto, el aparato conceptual aportado por Estany (1990) y las contribuciones de Field, Jardine, Smith y Kitcher a la reformulación de las teorias sobre la referencia pueden ayudar a dar cuenta, tanto de lo que supusieron las aportaciones de Lavoisier como las de Priestley y Cavendish. Al mismo tiempo permite una reconstrucción del proceso de la ciencia mucho más acorde con la historia real, en el sentido de que capta la gradualidad de dicho proceso.

Estas consideraciones poren en evidencia la necesidad de una nueva perspectiva en la explicación de la dinámica científica tal como proponíamos al principio de este trabajo. Por lo que se 
refiere a este caso histórico hemos visto que una forma de buscar parte de su microestructura ${ }^{9}$ es rastrear su desarrollo ontológico a partir de las teorías de la referencia que, en último término, son las que analizan filosóficamente el significado de los términos científicos. En este sentido los trabajos de Frege, Quine, Putnam, Kripke, Moulines etc. han sido de gran utilidad para esclarecer uno de los casos difíciles debido a la no extensionalidad del término "flogisto". Pero para ello hemos recurrido al contexto de la justificación, cuestión que la nueva perspectiva pone en primer plano de la explicación de la dinámica científica. Es decir, no puede abordarse la elección entre teorías en el progreso de la ciencia sin tener en cuenta el contexto de la justificación, entendiendo éste como lo que la filosofía de la ciencia ha dicho sobre estructura de las teorías científicas, sobre objetividad de la ciencia, sobre modelos de explicación científica, etc. La diferencia fundamental entre la mayoría de los modelos de cambio científico y la nueva perspectiva aquí presentada es que así como aquellos se presentan como modelos únicos, ésta funciona como un generador de modelos que aúnan los dos contextos cognitivos (justificación y descubrimiento) que, tradicionalmente, habían estado separados.

\section{BIBLIOGRAFIA}

CROSLAND, M.P. (1978) Historical Studies in the Language of Chemistry, New York: Dover Publications.

DONOVAN; A. (1988) "Lavoisier and the Origins of Modern Chemistry", QSIRIS, 2" serie, 4:214-231.

DonovaN, A., L. LALDAN, R. LAUDAN, ed. (1988) Scrutinizing Science. Empirical Studies of Scientific Change, Boston: Kluwer Academic Publishers.

ESTANY, A. (1990) Modelos de cambio científico, Barcelona: Crítica.

- (1993) Introducción a la filosofía de la ciencia. Barcelona: Crítica.

9 Otra forma de buscar la estructura fina o microestructura sería rastrear los cambios metodológicos. Para ello ver Estany (en prensa) "El papel de la metodologia en los modelos de cambio científico". 
- (1994) «El papel de las tipologias en la explicación de la dinámica científican, en Perspectivas actuales de lógica y filosofía de la ciencia, E. de Bustos, J.C. García-Bermejo, E. Pérez Sedeño, A. Rivadulla, J. Urrutia, J.L. Zofio (eds). Madrid: Siglo Veintiuno.

FIELD, H. (1973) "Theory Change and the Indetermination of Reference», Journal of Philosophy, 70: 462-481.

- (1974) "Quine and the Correspondence Theory", Philosophical Review, 83: 200-208.

FREGE, G. (1971) Estudios sobre Semántica, Barcelona: Ariel.

GRANDY, R. (1973) «Reference, Meaning and Belief» Journal of Philosophy, 70: $439-452$.

JARDINE, N. (1978) " Realistic" realism and the progress of science", en Action and Interpretation, ed. C. Hookway \& P. Pettit, 107-125, Cambridge.

KITCHER, P. (1993) The Advancement of Science Science without Legend, Objectivity without Illusions. New York: Oxford University Press.

KRIPKE, S. (1980) Naming and Necessity, Cambridge: Harvard University Pres.

KUHN,T. (1977) La estructura de las revoluciones científicas, Madrid: Fondo de Cultura Económica.

LAKATOS, I. (1983) La metodología de los programas de investigación científica, Madrid: Alianza.

LaudaN, L. (1978) Progress and its Problems. Towards a Theory of Scientific Growth, Los Angeles: University of California Press.

LAUDAN, L., A. DONOVAN et al. (1986) acientific Change: Philosophical Models and Historical Research" Synthese, 69, pp. 141-123, Dordrecht: Reidel.

LAVOISIER, A-L. (1743-1794) Oeuvres, París.

Moulines, U. (1992) Pluralidad y recursión. Estudios epistemológicos, Madrid: Alianza.

PRIESTLEY,J. (17759) Experiments and Observations on Different Kinds of Air, New York: Jhonson reprint 1970.

PUTNAM, H. (1973) «Meaning and Reference», Journal of Philosophy, 70: 699-711.

QUINE, W. (1969) Ontological Relativity and Other Assays, New York: Cabridge University Press.

SMITH, P. (1981) Realism and the Progress of Science, Cambridge: Cambridge University Press.

STAHL, G.E. (1718) Traité du Soufre, traducido del alemán por el barón de Holbach, reimpreso en 1966 , París.

THAGARD, P. (1988) Conceptual Revolutions, Princeton, Princeton University Press.

TOULMIN, S. (1977) La comprensión humana, Madrid: Alianza. 